\title{
Valoración de la eficiencia de remoción de arsénico en agua con carbón activado de jicaro sabanero (Crescentia alata) y su combinación con óxidos de bierro
}

\author{
Lic. Kathia Vanessa Rojas Cerda \\ Laboratorio de Contaminantes Metálicos \\ Centro para la Investigación en Recursos Acuáticos de \\ Nicaragua (CIRA/UNAN-Managua) \\ kathia.rojas@cira.unan.edu.ni
}

Fecha de recepción: 13 de febrero, 2020 / Fecha de aceptación: 20 de febrero, 2020

https://doi.org/10.5377/torreon.v9i24.9724

Palabras clave: Arsénico, carbón activado, óxidos de hierro, remoción

\section{RESUMEN}

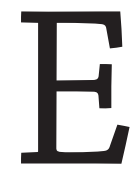

1 arsénico es una de las sustancias químicas más peligrosas en el mundo, según la Organización Mundial de la Salud, por su potencial de producir una serie de enfermedades desde diabetes y anemia hasta cánceres de pulmón y piel, entre otras. Por lo que es muy importante el desarrollar técnicas que permitan su remoción del agua para consumo humano. Este estudio evaluó la eficiencia de remoción de arsénico usando dos medios adsorbentes: carbón activado artesanal y óxidos de hierro en experimentos realizados por duplicado para carbón y por triplicado para el tratamiento secuencial de óxidos de hierro-carbón. Se utilizaron dos tamaños de carbón activado (2 - 4 y $0.6 \mathrm{~mm}$ ), dos tiempos de contacto (6 y 12 h) y tres concentraciones de trabajo (10, 25 y $50 \mu \mathrm{g}$ As/L). Los análisis de arsénico remanente en agua se realizaron mediante la metodología estándar internacional de absorción atómica por generación de hidruros. Los mayores porcentajes de remoción de arsénico se obtuvieron aplicando el tratamiento secuencial con óxidos de hierro y carbón activado, 54, 50 y 58 \%, 
respectivamente, para las tres concentraciones tratadas. El análisis de varianza realizado no detectó diferencias significativas entre los resultados obtenidos para 6 y 12 h de contacto, pero sí para los diferentes tamaños de carbón. Por lo que se recomienda usar el tiempo de contacto menor (6 h) y las partículas de carbón de menor tamaño $(0.6 \mathrm{~mm})$ para la remoción de Arsénico.

\section{INTRODUCCIÓN}

El arsénico (As) es una de las sustancias más peligrosas en el mundo, según la Organización Mundial de la Salud (OMS), por lo cual recomienda un valor máximo permisible para agua de consumo humano de $10 \mu \mathrm{g}$ As/L (OMS, 1995). Este metaloide se encuentra en las rocas de origen volcánico como la arsenopirita (FeAsS) y por el sistema de fallas geológicas pude lixiviarse al agua subterránea, siendo un peligro por su toxicidad y carcinogenicidad en humanos (Esparza, 2006).

En América Latina el arsénico ha causado problemas en la salud humana ya que un reporte dela ONU menciona a Argentina, Chiley México como países en los cuales se han encontrado aguas subterráneas contaminadas por arsénico. Diversos estudios correlacionan la ingesta de arsénico inorgánico con cáncer de pulmón, piel, vejiga y entre otros en órganos internos (Ferreccio, et al., 2000). También, existen varios efectos no cancerígenos relacionados a la ingesta de arsénico a bajas concentraciones, los cuales incluyen: enfermedades cardiovasculares, diabetes, anemia, desordenes reproductivos, inmunológicos, neurológicos y complicaciones en el embarazo, tales como el aborto y parto prematuro (Kapaj, Peterson, Liber, \& Bhattacharya, 2006).

En Nicaragua la presencia de niveles de arsénico superiores a los límites máximos permisibles en el agua de consumo humano fue detectada en 1996 en un pozo perforado del Zapote, Matagalpa, con concentración de 1320 mg As/L (UNICEF, 2002). Desde esa fecha el arsénico ha sido detectado en otras áreas del país, entre ellas se encuentras: la región de Kinuma en el Municipio de La Libertad, Chontales en la cual se registró un pozo con concentración de 106.7 $\mu \mathrm{g}$ As/L y en Cerro de Mina de Agua en el Municipio de Villanueva, Chinandega se registró un pozo con concentración de $12.5 \mu \mathrm{g}$ As/L (UNICEF, 2004). Además en Sébaco ubicado en el departamento de Matagalpa se detectaron concentraciones de arsénico en 21 pozos entre el rango 10 a $122 \mu \mathrm{g}$ As/L (Altamirano E, 2005).

Existen diferentes métodos para la remoción de arsénico tales como: procesos de precipitación incluyendo coagulación-filtración, filtración directa coagulación, floculación y ablandamiento (Han, Runnells, \& Wickramasinghe, 2002), procesos de intercambio aniónico (DeMarco, SenGruta, John, \& Greenleaf, 2003), filtración por membranas (USEPA, 2007) y procesos de adsorción que incluye la adsorción con sales de hierro (III) (Mohan \& Pittman, 2007). Este ultimo es un proceso de bajo costo y alta eficiencia muy utilizado para la remoción del arsénico, en el que este metal reacciona con la alcalinidad del agua para formar hidróxidos 
férricos, compuestos que forman pequeños conglomerados insolubles llamados "flóculos". El arsénico se liga a los flóculos formados de hidróxido férrico, que posteriormente son removidos por sedimentación y/o filtración en lechos granulares (Amirtharajah \& O’Melia, 1990).

Por otro lado, el carbón activado es muy empleado en procesos de adsorción de contaminantes (Universidad Politecnica de Sevilla, 2002) por lo que puede remover los flóculos de arsénico formados en el proceso de acomplejamiento con óxidos de hierro. La adsorción es un proceso por medio del cual se da la transferencia de moléculas disueltas en una fase líquida a una fase sólida en varias etapas sobre la superficie del carbón activado (Ngai, Dangol, Murcott, \& Shrestha, 2005).

Este estudio se basó en la valoración a escala de laboratorio de la eficiencia del carbón activado producido artesanalmente a base de cáscara de jícaro sabanero (Crescentia alata) y su combinación con óxidos de hierro para remover arsénico de agua.

\section{METODOLOGÍA}

\subsection{Diseño experimental}

Los ensayos para la determinación del tiempo y tamaño de carbón óptimos para la remoción de Arsénico se realizaron sometiendo tres concentraciones conocidas de As (10, 25 y $50 \mu \mathrm{g} \mathrm{As} / \mathrm{L}$ ) en agua desionizada preparadas a partir de una solución de $1000 \mu \mathrm{g}$ As/L diluida de una ampolleta Titrisol, dos tamaños de carbón activado (2-4 mm y $0.6 \mathrm{~mm}$ ) y durante dos tiempos de contacto (6 h y $12 \mathrm{~h}$ ). Estos ensayos se realizaron por duplicado en botellas plásticas de $355 \mathrm{~mL}$. La menor concentración ensayada $(10 \mu \mathrm{g} / \mathrm{L})$ se seleccionó ya que la OMS ha normado este valor como la cantidad máxima permisible de arsénico en agua para consumo humano y la máxima concentración ensayada (50 $\mu \mathrm{g}$ As/L) se seleccionó ya que se ha reportado que la exposición crónica a concentraciones similares está asociada a diversos padecimientos (Hughes, 2002).

Haciendo uso de los resultados obtenidos en los ensayos anteriores, el tiempo y tamaño de carbón activado en los cuales se obtuvieron los mejores porcentajes de remoción; se procedió a determinar los porcentajes de remoción de arsénico por medio de óxidos de hierro y carbón activado. Estos experimentos se realizaron con agua del pozo perforado de la comunidad de El Bonete, a la cual se le agregaron diferentes volúmenes de solución estándar de arsénico para obtener 14, 29 y $54 \mu \mathrm{g}$ As/L; respectivamente. Luego se procedió a colocar en contacto con los óxidos de hierro y el carbón activado producido artesanalmente a base de cáscara de jícaro (Crescentia alata) de $0.6 \mathrm{~mm}$ durante $6 \mathrm{~h}$. Estos ensayos se realizaron por triplicado.

El agua natural empleada se recolectó del pozo perforado con una profundidad de 100 pies, ubicado en la comunidad de El Bonete, municipio de Villanueva en el departamento 
de Chinandega, que presentó una concentración de 3,6 $\mu \mathrm{g}$ As/L y que tiene las siguientes características físico-químicas básicas: $\mathrm{pH}=7,37$, Conductividad eléctrica $=475 \mu \mathrm{S} / \mathrm{cm}$ y Dureza total $=220 \mathrm{mg} / \mathrm{L}$ (como $\mathrm{CaCO}_{3}$ ). La muestra fue colectada y preservada con $2 \mathrm{ml}$ de $\mathrm{HNO}_{3}$ por litro de muestra y se trasladó en un termo con hielo hasta el Laboratorio de Contaminantes Metálicos del CIRA/UNAN para su análisis, siguiendo el Protocolo de Cadena de Custodia del CIRA/UNAN (PROC-CM-02).

\subsubsection{Aplicación del carbón activado (2 - $4 \mathrm{~mm}$ y de $0.6 \mathrm{~mm}$ ) y dos tiempos de contacto ( 6 y $12 \mathrm{~h})$}

En este estudio se utilizaron embudos artesanales elaborados de botellas plásticas (355 $\mathrm{mL}$ ), seguidamente se pesaron $4 \mathrm{~g}$ de carbón activado de cáscara de jícaro (2-4 mm para cada embudo); luego se agregó $200 \mathrm{~mL}$ de cada una de las soluciones de As preparadas previamente (10, 25 y $50 \mu \mathrm{g}$ As/L, a cada embudo (se agito rápidamente con una varilla de vidrio). Transcurridas las $6 \mathrm{~h}$ de contacto se procedió a la filtración. Este mismo procedimiento se repitió para $12 \mathrm{~h}$ de contacto (se realizaron por duplicado). Se procedió a la determinación de las concentraciones finales del remanente líquido utilizando la técnica de generación hidruro.

Este mismo procedimiento se aplicó para los ensayos con el carbón activado con un diámetro $(0.6 \mathrm{~mm})$ a 6 y $12 \mathrm{~h}$ de contacto.

\subsubsection{Preparación de los clavos de hierro oxidados de media pulgada (1/2")}

Se colocaron los clavos de hierros no galvanizados de 1/2" pulgada en una pana con agua, dejándolos en contacto durante 2 semanas. Transcurridas las dos semanas se procedió a retirar los clavos del agua y se dejaron en el sol durante dos días, con el objetivo de eliminar cualquier partícula de agua (AMEC, 2015). Este procedimiento se realizó para producir óxidos de hierro, el que fue utilizado para retener arsénico.

\subsubsection{Determinación de la remoción de arsénico por medio de la remoción continúa con óxidos de hierro y carbón activado $(0.6 \mathrm{~mm}$ y $6 \mathrm{~h})$}

Se pesaron $4 \mathrm{~g}$ de clavos de $1 / 2$ pulgada previamente oxidados, los que se depositaron en cada embudo. Posteriormente se agregaron $200 \mathrm{~mL}$ de cada una de las soluciones con concentraciones de 14, 29 y $54 \mu \mathrm{g}$ As/L. Transcurridas 6 h de contacto, se procedió a separar el líquido, seguidamente se transfirieron a otros embudos, previamente preparados con $4 \mathrm{~g}$ de carbón activado $(0.6 \mathrm{~mm})$. Las soluciones se dejaron en contacto con el carbón activado durante 6 h. Transcurrido el tiempo de contacto, se procedió a la separación de la fase liquida para su posterior análisis (estas determinaciones se realizaron por triplicado).

Esta metodología fue tomada del filtro Kanchan desarrollado por investigadores del Instituto de Tecnología de Massachusetts (MIT), la Organización de Medio Ambiente y Salud 
Pública (ENPHO) de Nepal y el Programa de Apoyo al Suministro de Agua y Saneamiento Rural (RWSSSP) de Nepal, basado en filtros de filtración lenta e hidróxido de hierro (Ngai, Dangol, Murcott, \& Shrestha, 2005).

Una vez obtenidas las concentraciones finales de arsénico luego de ser sometidas durante un período de contacto con los medios adsorbentes, se procedió a calcular los porcentajes de remoción de arsénico por medio de carbón activado producido artesanalmente a base de cáscara de jícaro con tamaños de $2-4 \mathrm{~mm} ; 0.6 \mathrm{~mm}$ y a diferentes tiempos de contacto (6 y $12 \mathrm{~h}$ ):

Ecuación $N^{0}$ 1. Para calcular los porcentajes de remoción

$$
\% \text { de Remoción }=\frac{\mathrm{c}_{\mathrm{i}}-\mathrm{C}_{\mathrm{f}}}{\mathrm{C}_{\mathrm{i}}} \times 100 \%
$$

Donde:

$C_{\mathrm{i}}$ : Concentración inicial de As ( $\mu \mathrm{g}$ As/L)

$C_{\mathrm{f}}$ : Concentración final de As ( $\mu \mathrm{g}$ As/L)

\subsection{Descripción de la metodología analítica}

En el laboratorio de Contaminantes Metálicos del CIRA/UNAN Managua se determinaron las concentraciones de arsénico total a las muestras obtenidas en cada ensayo. La determinación se llevó a cabo por espectrometría de absorción atómica (espectrómetro Varian Spectr AA-240 FS) con la técnica generación de hidruro (VGA 77). El límite de detección del método es de 0.99 $\mu \mathrm{g}$ As/L para la matriz analizada (SMWW, 2005).

\section{RESULTADOS Y DISCUSIÓN}

\subsection{Remoción de arsénico transcurrido el tiempo de contacto de 6 y 12 h con carbón activado (2-4 mm)}

\subsubsection{Ensayo con tiempo de contacto de $6 \mathbf{h}$}

En el Figura 1 se muestran los promedios obtenidos de las concentraciones finales de arsénico de $10 \mu \mathrm{g}$ As/L, 24 y $50 \mu \mathrm{g}$ As/L (iniciales de 10, 25 y $50 \mu \mathrm{g}$ As/L), para un porcentaje de remoción de arsénico de $3 \%, 5$ y $0.5 \%$ respectivamente. 


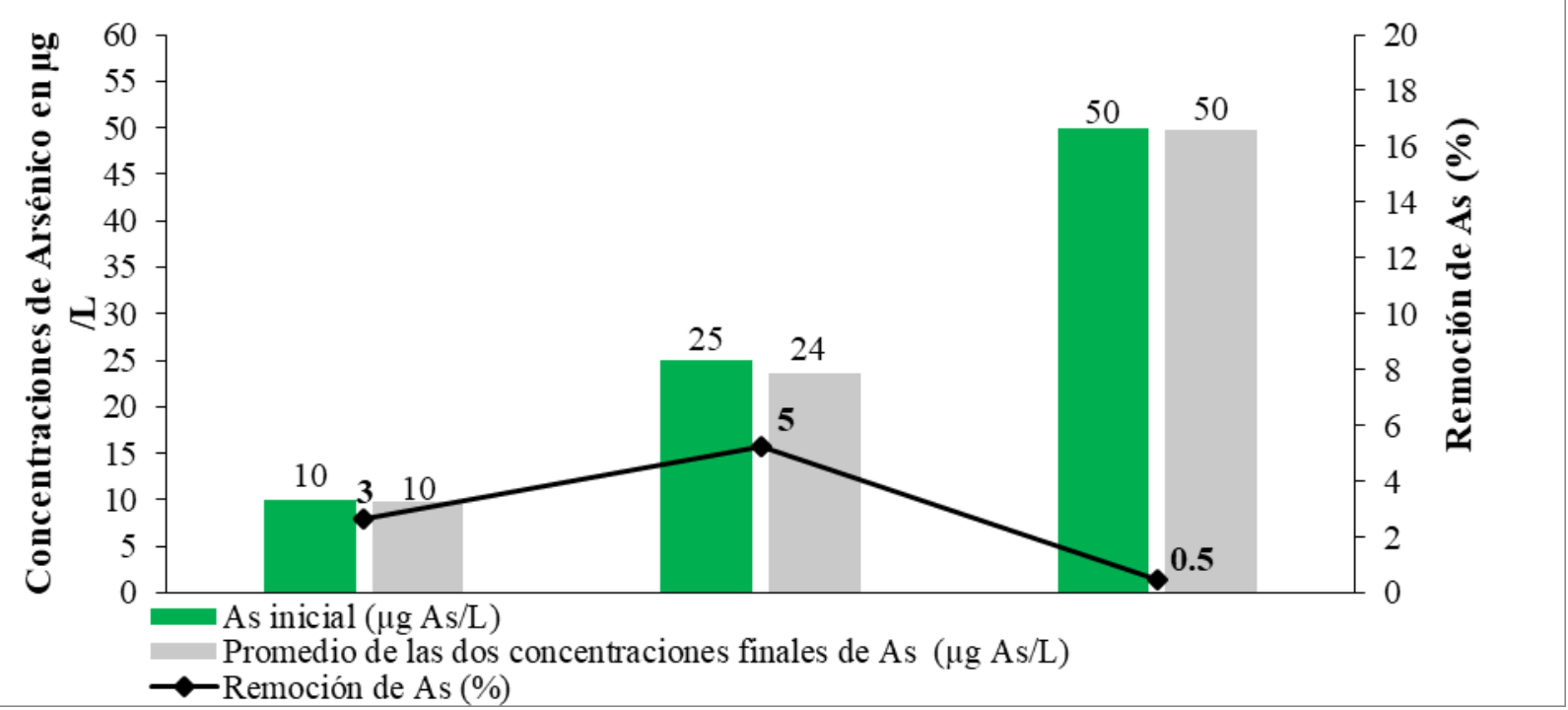

Figura 1. Concentraciones finales de As $(\mu \mathrm{g} / \mathrm{L})$ luego de ser expuestas durante 6 h de contacto con carbón activado de tamaño de 2-4 $\mathrm{mm}$.

Resultando la mayor remoción para la concentración de $25 \mu \mathrm{g}$ As/L y la menor para la de $50 \mu \mathrm{g}$ As/L, posiblemente a que los poros del carbón activado se saturaron disminuyendo el área de contacto entre el analito y el agente adsorbente. La capacidad de un carbón activado para retener una sustancia determinada no sólo está dada por su área superficial, sino por la proporción de poros cuyo tamaño sea el adecuado (Ures Rodríguez, Suárez López, \& Jácome Burgos, 2015).

\subsubsection{Ensayo con tiempo de contacto de $12 \mathrm{~h}$}

Los valores de concentración finales de remoción de Arsénico trascurrido el tiempo de contacto de $12 \mathrm{~h}$, analizadas bajo las mismas concentraciones previamente establecidas para los ensayos a 6 h, reflejaron resultados similares de remoción 9, 23 y $50 \mu \mathrm{g}$ As/L como se muestran en la figura 2. Los valores resultantes en porcentajes corresponden a 6,6 y $1 \%$ respectivamente, Siendo las mayores remociones obtenidas para las concentraciones de 10 y $25 \mu \mathrm{g} \mathrm{As} / \mathrm{L}$.

Basándose en los resultados obtenidos de los dos tiempos de contactos de 6 y 12 h, se aplicó el test de diferencias de medias, el cual permitió constatar que no existe diferencia significativa entre las concentraciones adsorbidas en los dos tiempos de contacto $(P>0.05)$. 


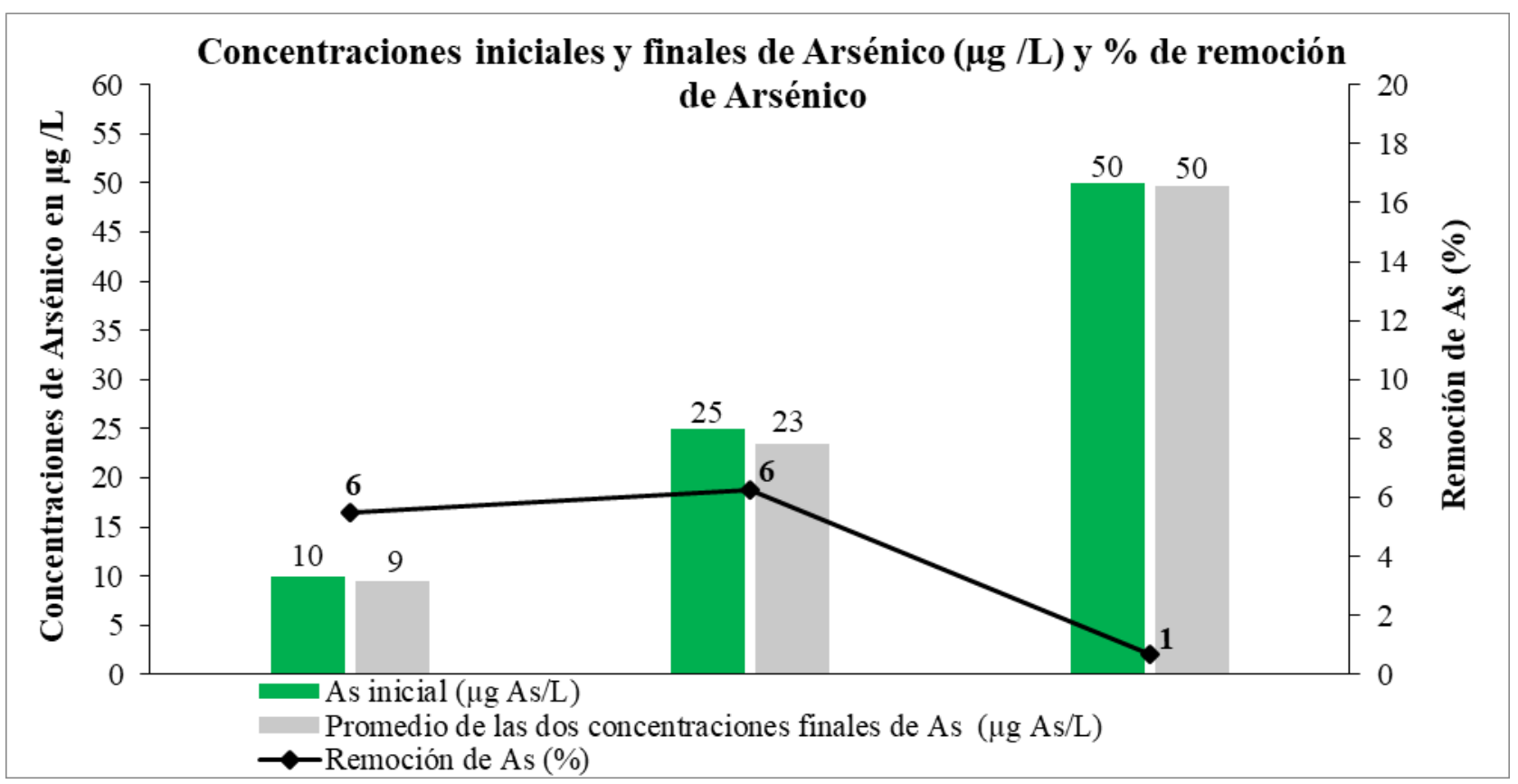

Figura 2. Concentraciones finales de As luego de ser expuestas durante $12 \mathrm{~h}$ de contacto con carbón activado de tamaño de 2-4 $\mathrm{mm}$.

\subsection{Remoción de arsénico transcurrido el tiempo de contacto de 6 y 12 h con carbón activado $(0.6 \mathrm{~mm})$ producido por $A D E C A B$}

\subsubsection{Ensayo con tiempo de contacto de $6 \mathrm{~h}$}

En general los resultados obtenidos para la remoción de arsénico empleando tres concentraciones diferentes (10, 25 y $50 \mu \mathrm{g}$ As/L), en 6 h de contacto con carbón activado 0.6 $\mathrm{mm}$, fueron mayores en comparación a los obtenidos con tamaño de 2 - $4 \mathrm{~mm}$, obteniendo concentraciones finales de 9, 23 y $43 \mu \mathrm{g}$ As/L, respectivamente (Figura 3). El mayor porcentaje de remoción se obtuvo a $50 \mu \mathrm{g}$ As/L con $14 \%$.

La baja adsorción del carbón activado quizás se deba al tamaño de sus poros, puede que en gran medida predominen los macroporos y mesoporos, puesto que esta es una propiedad muy importante y decisiva de los materiales adsorbentes es el tamaño de su poro, la mayor parte del área superficial total se encuentra en los microporos, por tanto, estos son los centros más disponibles a la adsorción para moléculas con relativamente bajo peso molecular (Ures Rodríguez, Suárez López, \& Jácome Burgos, 2015). 


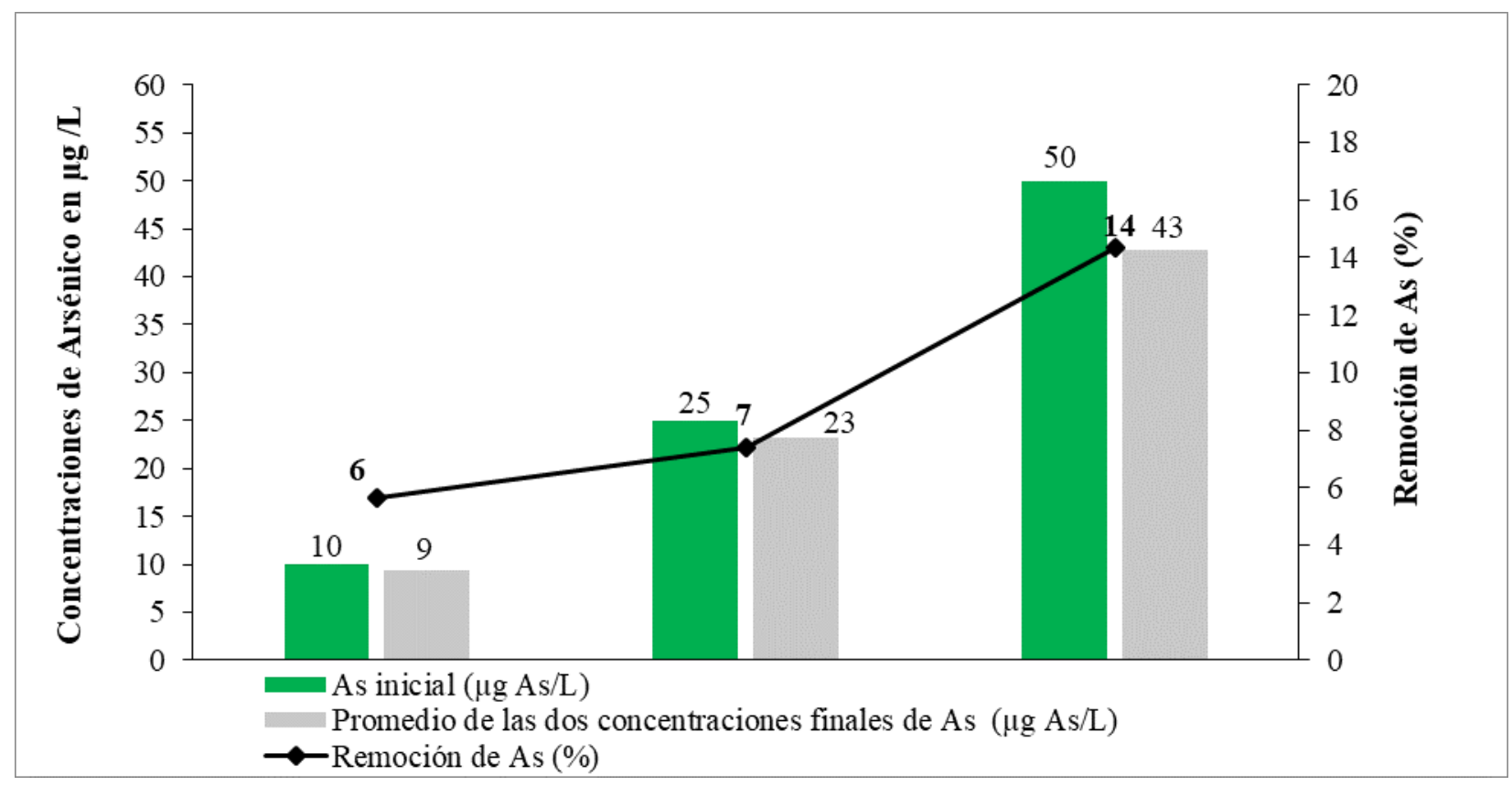

Figura 3. Concentraciones finales de As luego de ser expuestas durante 6 h de contacto en carbón activado con tamaño de $0.6 \mathrm{~mm}$.

\subsubsection{Ensayo con tiempo de contacto $12 \mathrm{~h}$}

Las concentraciones finales de las tres soluciones de arsénico ensayadas (10, 25 y $50 \mu \mathrm{g}$ As/L) al ser sometidas en contacto con carbón activado de $0.6 \mathrm{~mm}$ durante $12 \mathrm{~h}$, fueron 9 , 23 y 43 $\mu \mathrm{g}$ As/L (Figura 4). Los porcentajes de remoción corresponden a 7, 10 y 15 \%, respectivamente. Alcanzando el mayor porcentaje de remoción en la solución de $50 \mu \mathrm{g}$ As/L.

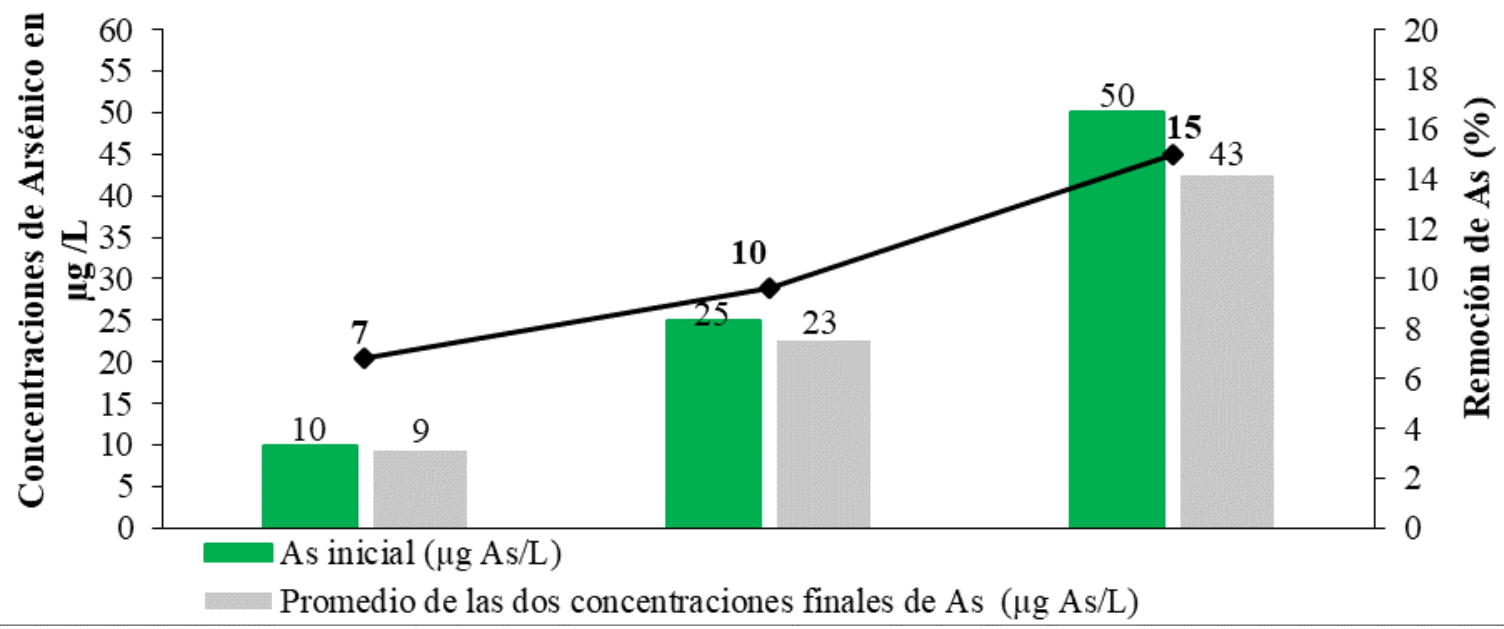

Figura 4. Concentraciones finales de As luego de ser expuestas durante 12 h de contacto en carbón activado con tamaño de $0.6 \mathrm{~mm}$.

En comparación con los tamaños del carbón activado, la capacidad de adsorción es mayor en $0.6 \mathrm{~mm}$, esto quizás se debe a que la adsorción se considera generalmente proporcional al 
área superficial específica de contacto, por tanto cuanto más finamente dividido y más porosos sea el carbón activado, mayor será el rendimiento esperado de adsorción por unidad de peso de adsorbente como indica (Ures Rodríguez, Suárez López, \& Jácome Burgos, 2015).

Ahora bien, el test de diferencias de medias, mostró que no existe diferencia significativa entre las concentraciones adsorbidas en los dos tiempos de contacto $P>0.05$, de manera que los tiempos de contacto no influyen significativamente en los porcentajes de remoción.

\subsection{Remoción de arsénico transcurridas 6 h de contacto con óxidos de hierro y carbón activado (0.6 $\mathrm{mm})$}

Las concentraciones finales de arsénico de las tres soluciones ensayadas con clavos de hierros oxidados (clavos de $1 / 2$ pulgada) y carbón activado artesanal de $0.6 \mathrm{~mm}(14,29$ y $54 \mu \mathrm{g}$ $\mathrm{As} / \mathrm{L}$ ) produjeron concentraciones finales de 6, 14 y $22 \mu \mathrm{g} \mathrm{As} / \mathrm{L}$, respectivamente (Figura 5).

En el tratamiento secuencial con óxidos de hierro y carbón activado se dio la mayor remoción de arsénico en la solución de mayor concentración (54 $\mu \mathrm{g}$ As/L) con 58 \%. Esta alta tasa de remoción pudo ser debida a una reacción de acomplejamiento superficial entre los óxidos de hierro y el arsénico formando así un flóculo, seguido por la adsorción de estos flóculos (Amirtharajah \& O’Melia, 1990), donde estas partículas de hierro cargadas con arsénico fueron retenidos por el carbón activado (Ngai, Dangol, Murcott, \& Shrestha, 2005).

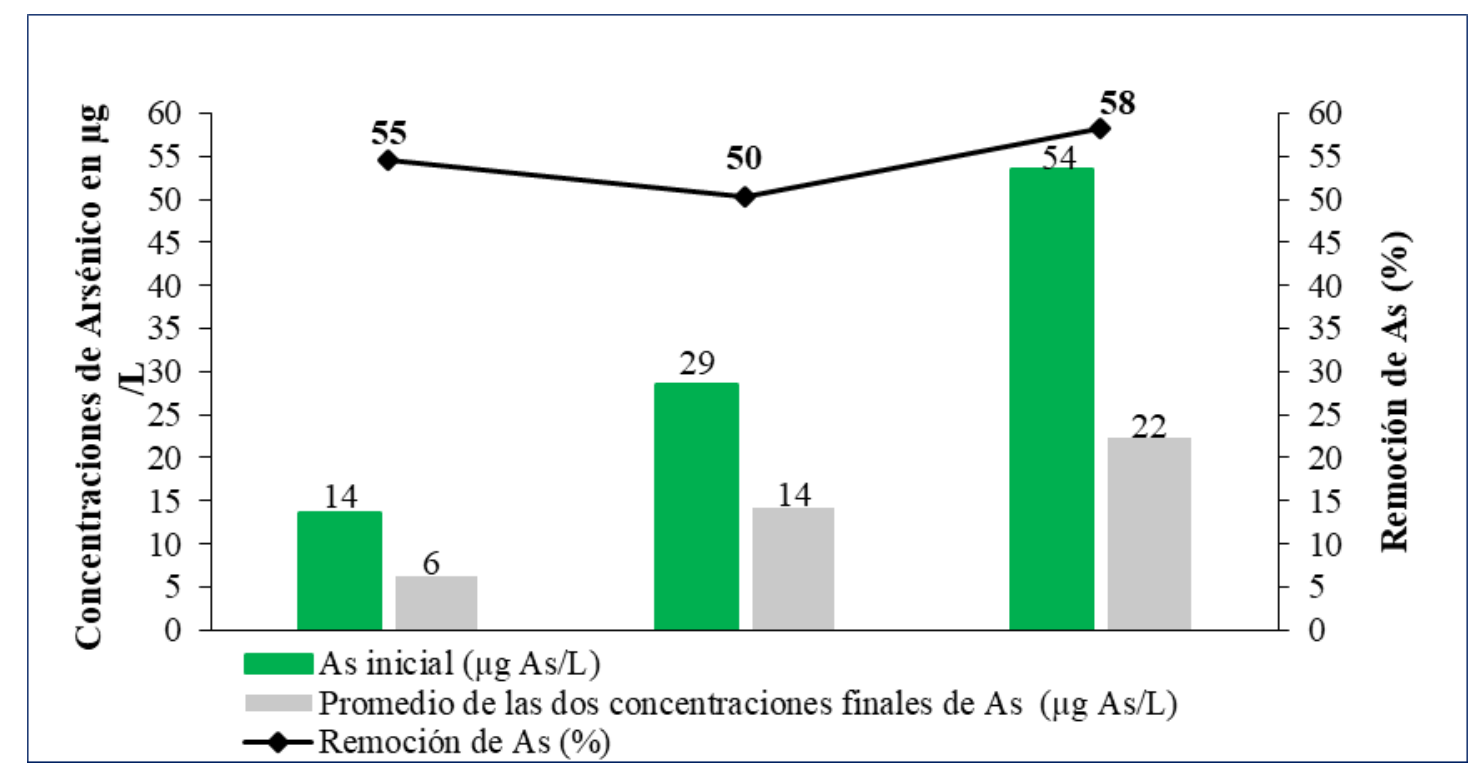

Figura 5. Concentraciones finales de As luego de someterse a filtración lenta por óxidos de hierro y luego expuestas durante 6 h de contacto carbón activado con tamaño de $0.6 \mathrm{~mm}$.

Las moléculas con mayor tamaño son adsorbidas sobre el carbón activado mejor que las moléculas de menor tamaño, esto debido a que la adsorción ocurre en los micros (tamaño inferior a $2 \mathrm{~nm}$ ) y mesoporos (2-50 nm) del carbón activado; siendo de mayor predominancia los mesoporos y es por ello que los flóculos se adsorben con mayor facilidad (Gregg \& Sing, 1982). 


\section{CONCLUSIONES}

Los mayores porcentajes de remoción de arsénico se obtuvieron para el tratamiento secuencial con óxidos de hierro y carbón activado artesanal de $0.6 \mathrm{~mm}$ y con un tiempo de 6 h de contacto (54, 50 y $58 \%$, respectivamente, para las tres concentraciones tratadas). No se detectaron diferencias significativas entre los resultados obtenidos durante los tiempos de contacto y tamaños de carbón activado.

\section{AGRADECIMIENTOS}

Al Fondo para Proyectos de Investigación (FPI), que otorga la Universidad Nacional Autónoma de Nicaragua (UNAN-Managua) y al Centro para la Investigación en Recursos Acuáticos de Nicaragua (CIRA/UNAN-Managua) por cofinanciar esta investigación.

\section{BIBLIOGRAFÍA}

Altamirano E, M. (2005). Distribución de la contaminación natural por arsénico en las aguas subterráneas de la subcuenca suroeste del Valle de Sebaco, Matagalpa-Nicaragua. Managua: CIRA/UNAN-Managua.

AMEC. (04 de Mayo de 2015). Componentes del filtro para la remoción de Arsénico. AMEC, Managua, Nicaragua.

Amirtharajah, A., \& O’Melia, C. (1990). Coagulation processes: Destabilization, mixing and flocculation (4th ed.). (F. W. Pontius, Ed.) Nueva York, N.Y., EE.UU: McGraw-Hill.

DeMarco, M. J., SenGruta, A. K., John, E., \& Greenleaf, J. E. (2003). Arsenic removal using a polymeric/inorganic hibrid sorbent. Water Research 37,. 164-176.

Esparza, M. C. (2006). Presencia de arsénico en el agua de bebida en América Latina y su efecto en la salud pública. International
Congress: Natural Arsenic in Groundwaters of Latin America. Mexico.

Ferreccio, C., González, C., Milosavjlevic, V., Marshall, G., Sacha, A. M., \& Smith, A. H. (2000). Lung cancer and arsenic concentrations in drinking water in Chile. Epidemiology. 11(6). 676-679.

Gregg, S. J., \& Sing, S. W. (1982). Adsorption, Surface Area and Porosity. London Academic Press.

Han, B., Runnells, T., \& Wickramasinghe, R. (2002). Arsenic renoval from drinking water by flocculation and microfiltration, Desalination 145. 293-298.

Hughes, M. (2002). Arsenic toxicity and potencial mechanisms of action. Toxicology letters. 133,, 1 - 16.

Kapaj, S., Peterson, H., Liber, K., \& Bhattacharya, P. (2006). Human Healh Effects from arsenic poisoning-a review. 
Journal of Environmental Science and Health. Part A 41,. 2399-2428.

Mohan, D., \& Pittman, ,. C. (2007). Arsenic removal from water/wastewater using adsorbents-a critical review. Journal of Hazardous Materials 142. 1-53.

Montero Campos, V., Quesada Kimsey, J., Ledezma Espinoza, A., \& Sandoval Mora, J. A. (2010). Determinación de arsénico en abastecimientos de agua para consumo humano de la provincia de Cartago, Costa Rica.

Ngai, T. K., Dangol, B., Murcott, S. E., \& Shrestha, R. R. (2005). Kanchan Arsenic Filter.Massachusetts Institute of Technology (MIT) and Environment and Public Health Organization (ENPHO). Kathmandu, Nepal. Kathmandu, Nepal.

OMS. (1995). Organizácion Mundial de la Salud.

SMWW. (2005). Standard Methods for the Examination of Water and Wastewater. American Public Healh Association (APHA), 21.
UNICEF. (2002). MONITOREO Y ATENCIÓN DE INTOXICADOS CON ARSÉNICOEN EL ZAPOTE, MUNICIPIO DE SAN ISIDRO, DEPARTAMENTO DE MATAGALPA, NICARAGUA 1994-2002. Managua: DRA. ALINA GÓMEZ C. Obtenido de https:// studylib.es/doc/6457827/monitoreoy-atenci\%C3\%B3n-de-intoxicados-conars\%C3\%A9nico-en-el-za...

UNICEF. (2004). Contribución al Estudio de Cinco Zonas Contaminadas Naturalmente por Arsénico en Nicaragua. Managua: UNICEF - Nicaragua.

Universidad Politecnica de Sevilla. (2002). MANUAL DEL CARBON ACTIVO. Recuperado el 2016, de http://www. elaguapotable.com/Manual\%20del\%20 carb\%C3\%B3n\%20activo.pdf

Ures Rodríguez, P., Suárez López, J., \& Jácome Burgos, A. (mayo de 2015). ADSORCIÓN EN CARBÓN ACTIVO (FTTER-002). INDITEX.

USEPA. (2007). Treatment Technologies for Arsenic Removal. 